\author{
Bánhegyi Mátyás ${ }^{1}$ - Nagy Judit ${ }^{2}$ \\ ${ }^{1}$ Budapest Business School University of Applied Sciences \\ College of Finance and Accountancy \\ Language Department of Finance and Accountancy \\ ${ }^{2}$ Károli Gáspár University of the Reformed Church in Hungary \\ Faculty of Humanities \\ Institute of English Studies
}

\title{
Solutions to Overcome Korean Students' Underperformance at Oral Presentation Tasks: Practical Tips for Hungarian Tertiary Settings
}

\author{
https://doi.org/10.48040/PL.2020.2
}

\begin{abstract}
Delivering oral presentations is a requirement in all academic programmes including studies in foreign educational contexts. Concerning American tertiary settings, Korean scholars identified numerous factors impacting the quality of Korean students' oral presentations. Based on the results of a previous qualitative case study conducted in Hungary, this paper identifies nine problematic areas in which Korean students experience difficulties in Hungarian tertiary settings. Four out of these nine areas have been selected for discussion in the present paper. Based on a practice-oriented literature review, this study outlines solutions, and also describes the authors' recommendations, to effectively combat these problems. The authors' concepts are made complete by the inclusion of author-designed worksheets and explanations concerning their use. It is envisaged that these proposed solutions and their discussion will contribute to developing Korean students' oral presentation skills in Hungarian tertiary-level educational contexts and will also benefit educators preparing other students for oral presentations.
\end{abstract}

Keywords: case study, in-class worksheets, oral presentation, Korean students, typical mistakes

\section{Introduction}

Korean students typically underperform in foreign educational settings when required to perform orally including giving an oral presentation. In order to research what factors contribute to this lower than desired performance at oral presentation tasks, Korean scholars examined American tertiary settings where Korean university students study: they identified numerous factors impacting the quality of Korean students' oral presentations. These factors include students' general language proficiency (Lee, 2009; Jeon, 2005; Kim, 2013); socio-cultural values and norms as well as educational practices and teaching methods different from those of the host culture (Liu, 2001; Lee, 2004; Shin, 2005; Shin, 2008; Lee, 2009; Kim, 2013); and specifics of the in-class learning environment (Tsui, 1996; Singelis et al., 1999; Kang, 2005; Shin, 2008; Lee, 2009).

As some Hungarian universities receive Korean exchange students and because these students wish to do their best to improve academic performance, it 
is also vital that Hungarian tertiary educators should be aware of the most typical mistakes Korean students make when they give oral presentations in Hungarian tertiary educational institutions. With a view to this, a qualitative case study was conducted in the scope of a research project between October 2014 and May 2017 (for a more detailed description of the research, see Bánhegyi and Nagy, 2018). The case study involved 28 Korean students coming from two Korean home universities, who took part in semester studies in Hungary and were hosted by the Károli Gáspár University of the Reformed Church in Hungary. The students studied in the English and American Studies BA Programme and participated in the "Advanced Language Practice" course. They typically studied in groups of 15-18, alongside with other international students from Poland, the Czech Republic, Germany, France, Spain and Portugal and their Hungarian peers. A requirement of successful course completion was to deliver a 15-minute-long oral presentation on any aspect of one of the topics covered during the course. Four dates announced at the beginning of the term were available for students' oral presentations, the so-called 'presentation sessions'. The evaluation of the digitally recorded presentations took place in writing, taking the following five assessment criteria into consideration: content (whether what the presenter was focusing on was congruent with the topic of the presentation); organization (whether the presenter observed the "introduction - statement of intention specific information - conclusion" pattern referred to below, and whether the presentation of information followed a logical structure); grammar (both accuracy and fluency); vocabulary (accuracy and fluency concerning both general and topic-specific items) and, finally, delivery (prosodics and non-verbal communication). The assessment criteria were presented during the orientation session to the course, and thus were known to the students prior to their presentations.

As a conclusion of the qualitative case study, nine areas have been identified where Korean students experience problems at Hungarian universities when preparing for and delivering oral presentations. These nine areas are as follows:

1. right choice of presentation topic

2. preparing a presentation outline

3. finding a clear and logical structure for the presentation (rather than deeming it an exercise in rhetoric/ stylistics)

4. reading out vs. delivering the presentation

5. using prompts and speaking notes to give the presentation

6. time management

7. altering the presentation on the spot

8. anticipating and preparing for the audience's questions

9. properly reacting to the audience's questions 
Motivated by the urge to offer tailor-made assistance to these students, in the scope of the present paper, the researchers conducted a literature review to explore what practical tips practising tertiary teachers recommend for combating the above problems. Based on these recommendations and the researchers' own experience, this study lists and explains the most commonly used and recommended methodological solutions. In addition, the paper also presents a few in-class activities that have been designed by the authors for the purpose of addressing each problem. For spatial constraints, this paper addresses issues 1-3 above. (It is envisaged that another two future papers will deal with issues 4-9 in detail.)

Before the discussion of the above-mentioned practical tips, however, a concise definition of oral presentation is given below, for the sake of clarity.

\section{Definition of oral presentation}

The literature offers numerous definitions of oral presentation. For this reason, in the context of this paper, a definition must be adopted that reflects the nature and the most common characteristics associated with oral presentations expected at BA level in English and American Studies. Based on this consideration and relying on Levin and Topping's definition (2006), an oral presentation is a preplanned genre that is practiced before it is given, and it is delivered on the spot to a specific audience. Price (1977) separates oral presentations into a general introduction (what the topic is, its relevance to the audience), a statement of intention (what the presenter wishes to achieve through giving the presentation), a part providing specific information (new and detailed information is provided to the audience), a conclusion (summary and lessons learnt), and an invitation for discussion (offering the audience the opportunity to ask questions, in the scope of which a meaningful thought exchange can develop).

Thus, in brief, in the context of the present paper, the term 'oral presentation' will be understood as a pre-planned, pre-practiced, typically intimidating oral genre, which is delivered on the spot to a specific audience and is composed of a general introduction, a statement of intention, a part providing specific information, a conclusion and an invitation for discussion.

\section{Literature review, recommended solutions and in-class worksheets}

\section{Right choice of presentation topic}

A right choice of topic appears to be decisive and thus fundamental concerning the success of the entire presentation and also functions as a crucial source of motivation for both presenters and their audiences. In their practical work focusing on good quality academic presentations, Bolster and Levrai (2014) warn that teachers should give the presenter a reason to present. This statement suggests that the topic in question should suit the presenters' interest and their 
level of cognitive capacity, just as it should also be motivating for the audience to listen to. At the same time, the topic should present a motive to speak and there should be content in it that presenters wish to share with their audiences. If that is not the case, even the most skilled presenter might fail. In addition, presenters should give the audience a reason to listen, Bolster and Levrai (2014) claim. This recommendation highlights that a good presenter chooses topics that are of interest and relevance to his/her audience and that benefit them in some way. Finally, Bolster and Levrai (2014) underline that content is by far the most important aspect of presentations and that style and rhetorical devices are only of secondary importance, which signals to presenters that the right choice of content is decisive when it comes to the success of their presentations.

International students in general - and Koreans are no exception - may be at a loss in terms of their choice of topic in the Hungarian tertiary setting due to differences in interests and cultures. Typically, Korean students would like to avoid sounding trivial, therefore they often choose an ambitious presentation topic the successful tackling of which would require a much higher level of language proficiency than their actual level of English. Out of the 28 students participating in the case study, 20 chose an abstract or highly abstract presentation topic. Out of these, the envisaged topic was above the language proficiency level of the given student (according to the Common European Framework of Reference for Languages, C1 or B2) in 14 cases, as attested by the resulting student presentation.

In addition, when it came to choosing their presentation topic, the case study participants seemed either to zoom in on something very particular (11 students), or they chose a very general topic and found it problematic to select aspects for forming the backbone of their presentation (5 students). In other words, narrowing down the topic to the couple of points the 15-minute time constraint would allow proved to be problematic in the case of dealing with wider subject areas.

Based on the findings of the literature and taking the above observations into consideration, we first and foremost suggest that the academics teaching a course where students are assigned to deliver similar oral presentations should take their time to personally advise Korean students on topics and perspectives suitable for their language level, interests and motivation. Instructors should be prepared to channel these students' interest towards those topics within the subject areas covered in class that are complex enough to maintain their interest and motivation, but, at the same time, are also congruent with students' linguistic abilities.

Furthermore, Korean students also need some training regarding the content a given title indicates. In this respect, the activity displayed in Figure 1 may prove useful. This activity invites students to consider six suggested presentation titles and predicts the potential problems each title and the underlying topic may entail. With the help of these concrete examples, it will be 
easier for students to understand the implications of the chosen title for the content of the presentation.

Figure 1. Oral Presentation Task Sheet - Task 1: Presentation Title

\section{Presentation title}

The following titles may be problematic for your presentation. Can you guess why?
a) Esztergom
b) Esztergom - A brief history
c) History of Esztergom's name and its coat-of-arms
d) Szendrey-Karper László Guitar Festival
e) Esztergom Panoptikum: Theatre, Music and Wax Figures
f) Esztergom Castle Hill Research Project

To explain the implications mentioned above, in the first case, the title "Esztergom" encapsulates far too many aspects to talk about during a 15-minutelong presentation. In the second case, a sub-title has been added ("Esztergom A brief history") to focalise the presentation; however, the hinted subtopic is still too wide and not informative enough of the actual content of the presentation. The title "History of Esztergom's name and its coat-of-arms" does not fit the topic-subtopic focus pattern, and the subtitle is awkwardly chosen: it creates the impression of a random choice. Next, "Szendrey-Karper László Guitar Festival" is far too specific to fill a 15-minute-long presentation. In such cases, if students are invited to come up with possible subpoints for the topic addressed in the title, they will self-discover the overly specific nature of their chosen title. The assigned content within the title "Esztergom Panoptikum: Theatre, Music and Wax Figures" seems rather arbitrarily selected and sequenced. Finally, "Esztergom Castle Hill Research Project" is suggestive of a very challenging presentation content, which, in addition, is also quite unlikely to generate interest in the audience. By discussing the implications of the titles for presentation topics and subtopics, Korean students will gain a better understanding of their choice of presentation title (and topic).

\section{Preparing a presentation outline}

It is a typical mistake regarding Korean students' oral presentations that the content does not come through because students do not prepare a real outline, and thus are not clearly aware of the structure of their presentation. Moreover, it also follows from the lack of their outline that they cannot communicate their presentation content clearly to their audiences to facilitate their understanding of the content (Bolster - Levrai, 2014).

It is also a common mistake that students focus on the final outcome of their oral presentations, i.e. the final version to be communicated in class, and fail to go through the steps leading up to the completed presentation. This way their presentation may contain logical discrepancies, inaccuracies, aspects that do 
not belong there, topics falling outside the scope of their presentations. Regarding this problem the literature suggests that students should choose one section of their presentation and present it to different people so that the presentation evolves over time (Bolster - Levrai, 2014). This provides students with feedback from different audiences concerning the content, the scope and the logic of their presentations.

The third typical problem related to preparing an outline the literature mentions is that, in their presentations, students want to include everything and every aspect of the topic in question that they have researched while preparing for their talks. This usually results in an unclear outline structure. In order to overcome this problem, Edwards (2013) suggests selecting only the core arguments for the given presentation and leaving all subsidiary arguments for another later presentation.

To overcome the challenge of outline preparation successfully, a process model should be employed, which guides students from their initial ideas to the actual outline. Such an approach is crucial in the case of Korean students as they may be used to different organizing principles internalised during their prior schooling (e.g. writing sermon-like speeches to be read out, where aesthetic principles override the rules and the logic of point-subpoint organization). Then, once the process model has been completed, model outlines should also be presented to help students achieve structural balance in their subpoints. In addition, when students have been advised on their title (see discussion in point 1 above), completed the process model and have analysed several model outlines, they will also need assistance with finding a logically sound structure within their own topic.

Figure 2 displays a sample activity which introduces the stage of the process model, in which some relevant aspects of the given topic (ghost towns) have already been selected to start students off.

Figure 2. Oral Presentation Task Sheet - Task 2: Presentation Topic

\section{Preparing an outline}

How would you arrange the following information resulting from brainstorming into a logical outline on Ghost Towns?

1) activities: mine tours, gunfight stunt shows, gold panning, restaurants, Mystery Shack, historic railroad, trinket stores

2) "a town for which the reason for being no longer exists" (T. Lindsey Baker)

3) Calico was founded in 1881.

4) economic activity shifting elsewhere

5) Chernobyl and Pripyat: disaster-stricken area

6) It became an Official Silver Rush Ghost Town in 2005.

How would you supplement this information for preparing an outline of a presentation on this topic? 
At this stage, students are first expected to arrange the existing information into a logical structure, then supplement the given content to produce a full outline through Internet research. As for the suggested solutions for this activity, the quotation in (2) may form part of the introduction, following a descriptive definition. This section can conclude with some well-known examples and their pictorial illustration. Figure 3 shows a potential arrangement of the subpoints in the body of the outline as well as additional information to supplement these points with (in red).

Figure 3. Oral Presentation Task Sheet - Task 2: Presentation Topic - Outline body solutions

\author{
Body: \\ a) Reasons for abandonment (4), (5) [depleted natural resources, railroads and roads bypassing \\ but no longer accessing town, human intervention (e.g. relocation), wars] \\ b) One particular example: \\ Calico $\rightarrow$ \\ 1. past (3) [Silver King Mine, California's largest silver producer in the 1880 s, abandoned by \\ 1907, revival attempt: 1915, becomes a County Regional Park], \\ 2. present (6), (1) [place restored to look like the Calico of the 1880 s, more details on the \\ features enumerated in (1), special events throughout the year]
}

And, to conclude the outline, recommending further reading or relevant footage, some thoughts on the future of ghost towns or some ghost town-related fun facts may be added. Guiding students through this example may help them understand the entire process of producing a carefully-designed outline.

\title{
Finding a clear and logical structure for the presentation
}

As point 2 has already hinted, many Korean students believe that an oral presentation is primarily an exercise in rhetoric or in stylistics, and fail to bear in mind that the primary aim of an academic presentation is to convey information. An approach of this kind may result in a presentation with faltering logic or may result in a logical structure that is not properly exposed or is not understandable to the audience. Concerning the expectation to effortlessly understand a good oral presentation, Hindley and Roberts (2013) claim that a good oral presentation will speak to the point, features structured argumentation and presentation of ideas, is rich in digestible information content and relies on sources including the literature and/or research data.

Furthermore, concerning the content and also the structure of presentations, Bolster and Levrai (2013) argue that academic oral presentations demonstrate mastery of the subject, contain research-based arguments with verifiable and referenced information and sources, feature a logically arranged synthesis of sources, and encourage further discussion. Students must be warned that their presentations should exhibit the above features and these should be taught and practised. 
With respect to genre-specific vocabulary and its importance to helping the audience follow the presentation, Grussendorf (2007) draws attention to signalling devices that help the audience differentiate between different parts of the presentation and also aid the audience in following the logic of the talk. Minimally, the three parts of the presentation (introduction, body and conclusion) and also the invitation to discussion should be indicated by using appropriate lexical items such as "I'll start off by..." Moreover, it is not enough to teach these vocabulary items to the students, they should be practiced and their use must also be encouraged and expected.

Based on the above, it must be explained to students with a Korean educational background that a structurally simple and logically clear presentation (introduction - body - conclusion) does not signal superficiality or second-rate content whereas structural complexity or overly abstract content may be an impediment in the way of an audience-friendly presentation. To enhance students' better understanding of what an audience-friendly presentation structure looks like, structurally appropriate presentation models must be provided and explained in detail. Last but not least, signalling devices and their appropriate use must be covered in class to guide the audience through the presentation.

As for presentation models, providing structurally impaired models can also facilitate students' understanding of right presentation structures. Figure 4 displays two presentation sketches with structural faults. Students are given some time to reflect upon the potential problems with these sketches, point them out and try to mend them.

Figure 4. Oral Presentation Task Sheet - Task 3: Finding a Clear and Logical Structure for the Presentation

a)

\section{Natural tourist sites}

Telkibánya can be found in the upper northern part of Borsod-Abaúj Zemplén County; located 20 kilometres away from the Hungarian -Slovak border. It is part of the Zemplén protection area ${ }^{1}$ as well.

Forest and mine: nearby forest which is home to many of the region's mines. Not only can you go and search for various quartz and half-precious stones local to the region (including opal, white quartz and rose quartz), but you can also dig them up yourself.

Cser-hill perlite pit²: perlite is a volcanic rock which was mined in the past.

Ice-cave: it is not a regular cave, however, rather an exploratory audit ${ }^{3}$. The rock of Király Hill is of rhyodacite ${ }^{4}$ which rock easily breaks up by frost, the fissures will fill up with water from melting snow and precipitation which freezes over in winter. Because of this ice hidden in the fissures, the yearly average temperature of the hill is $10-15$ Clower than measured in the valley soil. 
b)

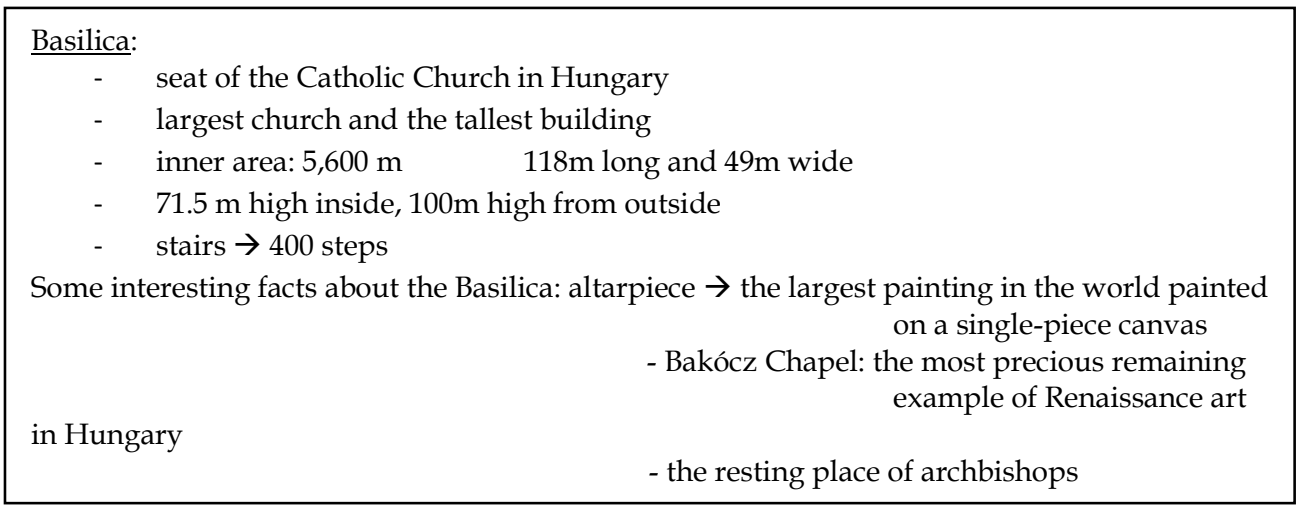

The problems the sketches demonstrate draw both on form and contentrelated issues. As for the concrete examples in a) and b), the presentation point "Natural Tourist Sites" contains full sentences and it is not divided into real subpoints, which will make it difficult to follow. In addition, more information could be included on what a "landscape protection area" actually is. The point also contains highly specific vocabulary items, which, on the one hand, will make it difficult for the presenter to remember the terminology to be used during the talk and, on the other hand, will cause difficulties for the audience to understand the presentation. What regards "Basilica", form is the dominant problem here: the presented information is scattered all over the page within the point, and the subpoint structure is fuzzy.

Further typical issues worth being covered through similar 'faulty' presentation points and sketches as demonstrated through the examples a) and b) include ornate vocabulary, the inappropriate use of jargon, irrelevant information, simplification of content, inappropriate elaboration, pointless addition or omission of information, in-depth versus shallow discussion.

\section{Conclusions}

Using the results of a previous qualitative case study conducted at a Hungarian university, this paper identified nine problematic areas where Korean students experience difficulties in Hungarian tertiary classes. Out of these nine areas, four were selected for discussion in the present paper. Drawing the literature and the authors' methodological experience and practice, numerous suggestions were made as to the development of Korean students' oral presentation skills.

As a final word, it is worthwhile to stress that being aware of the educational background of foreign students helps educators offer tailor-made solutions specifically targeting the student population in question. Such diversification of methodology seems to prove helpful for students with different educational backgrounds. Without doubt the above methodological ideas and 
their descriptions can be used for successfully preparing international students for oral presentations in the Hungarian tertiary educational setting.

\section{References}

Bánhegyi, M. - Nagy, J. (2018): Successful Oral Presentations in Business English: A Case Study of Korean Students. In: Bocz Zs. - Besznyák, R. (eds.) (2018): Porta Lingua 2018. Szaknyelvoktatók és -Kutatók Országos Egyesülete: Budapest. 205-220

Bolster, A. - Levrai, P. (2013): Developing Research-Based Academic Oral Presentation Materials. Paper delivered at: Materials Development Association Conference. Liverpool

Bolster, A. - Levrai, P. (2014): Substance over Style: Meaningful Academic Oral Presentations. Paper delivered at: The Sixth Centre Language Studies International Conference of the National University of Singapore. Singapore

Edwards, L. (2013): How to do a brilliant Presentation. The University of Hong Kong, School of Modern Languages and Culture. Lecture Notes

Grussendorf, M. (2007): English for Presentations. Oxford University Press: Oxford.

Hindley, C. - Roberts, D. (2013): Business and Academic skills - incompatible or complementary? Paper delivered at: IATEFL BESIG Conference. Prague

Jeon, J. (2005): A Study on Oral Presentation Anxiety and Confidence: A Comparison between L1 and L2 Presentations. The Journal of Asia TEFL. 2/2. 89-115.

Kang, S. J. (2005): Dynamic emergence of situational willingness to communicate in a second language. System. 33. 277-292. DOI: https://doi.org/10.1016/j.system.2004.10.004

Kim, J. (2013): Oral Communication Needs of New Korean Students in a US Business Communication Classroom. Global Business Languages. 18.

Lee, G. (2009): Speaking up: Six Korean students' oral participation in class discussions in US graduate seminars. English for Specific Purposes. 28. 142-156. DOI: https://doi.org/10.1016/j.esp.2009.01.007

Lee, K. S. (2004): Cultural learning and linguistic characteristics of Korean students in the United States: Perceptions of professors and students. Fordham University: New York

Levin, P. - Topping, G. (2006): Perfect presentations. Open University Press: Berkshire, UK

Liu, J. (2001): Asian students' classroom communication patterns in U.S. universities: An emic perspective. Ablex: Westport, CT

Price, J. E. (1977): Study skills - with special reference to seminal strategies and one aspect of academic writing. In: Holden, S. (ed.) (1977): English for Specific Purposes. Modern English Publications: London. 26-29

Shin, H. (2005): Exploring the possibilities for EFL critical pedagogy in Korea - a two-part case study. Critical Inquiry in Language Studies: An International Journal. 2/2. 113-138. DOI: https://doi.org/10.1207/s15427595cils0202_3

Shin, I. (2008): Necessary Skills in English for Korean Postgraduate Engineering Students in London. Educate - Special London Issue. 50-61

Singelis, T. M. - Bond, M. H. - Sharkey, W. F. - Lai, C. Y. S. (1999): Unpackaging culture's influence on self-esteem and embarrassability: The role of self-construals. Journal of Cross-Cultural Psychology. 30. 315-341. DOI: https://doi.org/10.1177/0022022199030003003

Tsui, A. (1996): Reticence and anxiety in second language learning. In: K. Bailey and D. Nunan (eds.) (1996): Voices from the language classroom. Cambridge University Press: Cambridge, UK. 145-167 